\title{
Three-dimensional optical beam propagation and solitons in photorefractive crystals
}

\author{
Bruno Crosignani and Paolo Di Porto \\ Dipartimento di Fisica, Università dell'Aquila, L’Aquila, Italy, \\ and Istituto Nazionale di Fisica della Materia, Unità di Roma I, Rome, Italy \\ Antonio Degasperis \\ Dipartimento di Fisica, Università di Roma "La Sapienza," P. le Aldo Moro 2, 00185 Rome, Italy, \\ and Istituto Nazionale di Fisica Nucleare, Sezione di Roma I, Rome, Italy \\ Mordechai Segev \\ Department of Electrical Engineering and Center for Photonics and Optoelectronics Materials, Princeton University, \\ Princeton, New Jersey 08544 \\ Stefano Trillo \\ Fondazione Ugo Bordoni, Via B. Castiglione 59, 00142 Rome, Italy
}

Received January 31, 1997; revised manuscript received June 16, 1997

The model equations for beam propagation in photorefractive material are simplified under appropriate conditions. The possibility of obtaining bright and dark screening soliton solutions in $2+1$ dimensions is investigated, and, whenever possible, their amplitude-size relation is displayed. (c) 1997 Optical Society of America [S0740-3224(97)02511-3]

PACS numbers: $\quad 42.50 . \mathrm{Lc}, 42.50 . \mathrm{Dv}, 05.30 . \mathrm{Jp}, 42.65 . \mathrm{Ky}$.

\section{INTRODUCTION}

Self-trapping of optical beams has been extensively studied during the past three decades. It occurs when diffraction is exactly balanced by an optical nonlinearity, leading to the formation of solitary waves. Although selftrapping was first proposed in ideal Kerr media ${ }^{1}$ described by the nonlinear Schrödinger equation that guarantees that the solitary waves are strictly stable solitons in two dimensions (i.e., $1+1 \mathrm{D}$ ), ${ }^{2}$ it was demonstrated experimentally in nonideal Kerr media such as gases, ${ }^{3}$ fluids, ${ }^{4}$ and solids. ${ }^{5}$ One of the most distinctive properties of Kerr-type solitons is their instability in systems with dimensionality greater than or equal to 3 . This includes either instability of cw beams trapped in both transverse dimensions (i.e., $2+1$-D case), ${ }^{2}$ which leads to catastrophic self-focusing ${ }^{6}$ and eventually to beam breakup, or instability of a one-dimensional (i.e., 1 +1 -D) soliton in bulk media, which leads to beam breakup owing to transverse instability. ${ }^{7}$ Self-trapped optical beams in other non-Kerr, nonlinear media have also been investigated. Theoretical studies have shown the existence of solitary waves in saturable nonlinear media ${ }^{8-13}$ which should permit stable $2+1-\mathrm{D}$ selftrapping. These studies were followed by recent experiments that reported on collision and fusion of such solitons. ${ }^{14}$ A different type of nonlinearity that allows for $2+1$-D self-trapping is based on $\chi^{(2)}$ parametric amplification effects, in which beams at fundamental and second-harmonic frequencies interact and trap one another. $^{15}$

Photorefractive (PR) spatial solitons ${ }^{16-46}$ are based on an optical nonlinearity that is different from all those mentioned above, namely, the PR effect. It results from charge excitation, transport, and trapping, which give rise to a space-charge field that in turn modulates the refractive index by means of the electro-optic (i.e., Pockels) effect. $^{47,48}$ By and large, the properties of all PR spatial solitons differ greatly from those of other solitons as manifested in their stable self-trapping in both transverse dimensions and their existence at microwatt and lower power levels. In fact, PR solitons were the first optical solitons observed to be self-trapped in both transverse dimensions in a bulk material. ${ }^{19-22,29-32}$ At present, three generic types of PR soliton are known: quasi-steadystate, ${ }^{16-23}$ screening, ${ }^{24-27,29-33,35-43}$ and photovoltaic ${ }^{44,45}$ solitons. Quasi-steady-state solitons were discovered first. ${ }^{16,19}$ They exist during the slow screening process of an external field applied to a PR crystal. Screening solitons exist in the same media but in steady state when the field is nonuniformly screened. ${ }^{24,29}$ Photovoltaic solitons exist in PR materials with a strong photovoltaic current (e.g., $\mathrm{LiNbO}_{3}$ ) and use the refractive-index perturbation associated with a photovoltaic field to guide and confine the soliton. ${ }^{4,45}$ Recently, vector solitons ${ }^{38}$ and vector soliton interactions ${ }^{25,40,41}$ were also predicted, and coupled soliton pairs were predicted ${ }^{42}$ and observed. ${ }^{43}$ Of 
all these types of $\mathrm{PR}$ soliton, the screening soliton can be visualized intuitively in the following manner. A narrow light beam propagates in the center of a biased dielectric medium. As a result, in the illuminated region the conductivity increases and the resistivity decreases. Therefore the voltage drops primarily in the dark regions, and this leads to a large space-charge field there. The refractive index changes in proportion to the space-charge field with opposite (i.e., negative) sign. Thus, in the dark regions surrounding the beam, a large negative index change creates an effective graded-index waveguide that guides the beam that generated it, in a self-consistent manner (see, e.g., Fig. 1 of Ref. 27).

Interestingly enough, all types of PR soliton were first predicted theoretically, ${ }^{16,24,44}$ and only then was an experimental observation made. ${ }^{19,29,45}$ However, existing theories of PR solitons describe only $1+1$-D solitons and hence cannot explain the experimental observations of self-trapping in both transverse dimensions. A first attempt to provide a theoretical model for $2+1$-D PR solitons was made in Ref. 49. However, these results, besides being essentially of a numerical nature, are not able to account for the experimental observation of a selftrapped propagating beam maintaining an axially symmetric invariant diameter. A possible reason for this discrepancy is associated with the fact that solitons are characterized by an existence curve (the set of parameters that can support soliton) that relates the soliton's width and its peak amplitude to the optical wavelength in the medium and the material nonlinearity. The existence curve is typically a single-valued function. Small deviations from the soliton existence curve can be tolerated and suppressed by the soliton's stability features, but large deviations lead to nonstationary evolution. Thus it is extremely difficult to find solitons by use of numerical simulations alone, without knowing the soliton existence curve, simply because one needs to perform simulations that span the entire parameter space. To illustrate this issue we draw on the extensively studied case of Kerrtype solitons, for which an analytical solution is well known; ${ }^{1,2}$ several experimental observations were reported (e.g., Ref. 5). For $1+1$-D Kerr-type solitons, Burak and Nasalski ${ }^{50}$ have shown that an input Gaussian beam converges to a soliton only if its parameters are close enough to the soliton parameters (i.e., to the existence curve). In all other cases the propagating beam is characterized by oscillations in its diameter and does not converge to a soliton. Similar studies were performed specifically on $1+1-\mathrm{D}$ PR solitons ${ }^{25}$ and have shown a similar trend. It is therefore not surprising that purely numerical calculations with arbitrary beam and nonlinearity parameters have failed to find even the $1+1$-D $\mathrm{PR}$ solitons $^{26}$ for which both analytical and experimental $^{24-27,33,43}$ results have been reported. The arbitrarily chosen parameters for this calculation were simply too far from the existence curve. At this point it is obvious that an analytical approach to searching for 2 +1 -D PR solitons is necessary if one is to find at least the soliton existence curve so that the parameters suitable for soliton propagation can be identified.

In this paper we present a theory of three-dimensional propagation in biased PR media and analyze the specific case of screening (i.e., steady-state) solitons. We highlight the differences between the two-dimensional and the three-dimensional cases. For the latter case we derive a system of two coupled equations that govern the evolution of the optical field and the potential of the space-charge field. Under the appropriate conditions the equation that relates the space-charge field $\mathbf{E}$ to the optical intensity is formally linear. In this regime, with the further condition that the maximum normalized intensity be smaller than unity, we derive a single integrodifferential equation that governs the nonlinear propagation of the optical field inside the PR crystal. Under suitable conditions, that is, whenever the nonlocal contributions are neglected, this equation admits of solutions that correspond to axially symmetric bright and dark solitons. We determine the width-amplitude relations that have to be satisfied to ensure their existence, namely, the soliton existence curve. Moreover, in the significant case in which a comparison with current experiments is possible, we are able, for the dark solitons, to work out a simple way of deriving the existence curve. Finally, in the $1+1-\mathrm{D}$ case, we provide general results on the temporal evolution to steady state of the space-charge field.

\section{OPTICAL PROPAGATION IN PHOTOREFRACTIVE CRYSTALS}

We review the general formalism that allows us to describe the propagation of an optical beam inside a PR crystal (Fig. 1).

The main mechanism that affects beam propagation is the nonlinear refractive-index variation associated, through the Pockels effect, with the onset of a static electric field $\mathbf{E}$ inside the crystal (PR effect). The first task is thus to evaluate this space-charge field $\mathbf{E}$ as a function of the crystal parameters and of the intensity of the propagating beam. To this end we rely on the usual model, ${ }^{48}$ according to which the PR effect is described by the following set of equations:

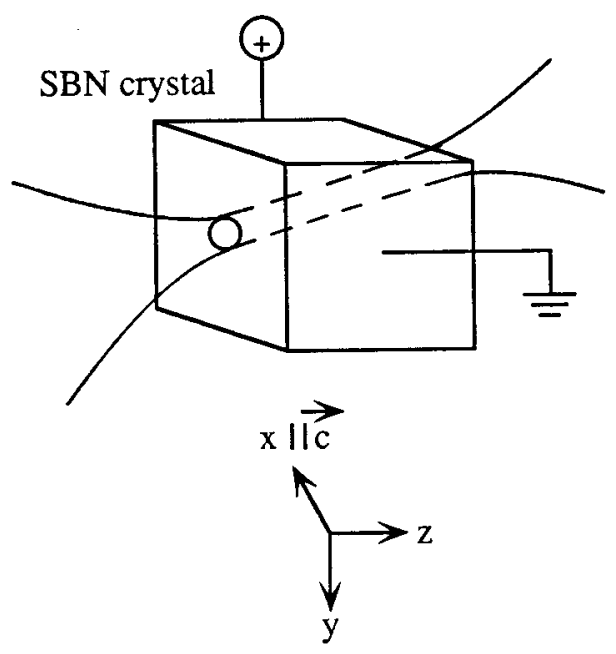

Fig. 1. Geometry of propagation in the PR crystal. 


$$
\begin{aligned}
\frac{\partial}{\partial t} N_{D}^{+} & =(\beta+s I)\left(N_{D}-N_{D}^{+}\right)-\gamma N_{e} N_{D}{ }^{+}, \\
\nabla \cdot(\tilde{\epsilon} \mathbf{E}) & =\rho, \\
\frac{\partial}{\partial t} \rho+\nabla \cdot \mathbf{J} & =0, \\
\rho & =q\left(N_{D}^{+}-N_{A}-N_{e}\right), \\
\mathbf{J} & =q \mu N_{e} \mathbf{E}+\mu K_{B} T \nabla N_{e},
\end{aligned}
$$

where $I=\left|\mathbf{E}_{\text {opt }}\right|^{2}$ is the optical intensity, $\mathbf{E}$ is the spacecharge field, $N_{D}$ is the density of donors, $N_{D}{ }^{+}$is the density of ionized donors, $N_{A}$ is the density of acceptors, $N_{e}$ is the electron density, $\beta$ is the dark-generation rate, $s$ is the photoionization cross section, $\gamma$ is the recombination rate coefficient, $\mu$ is the electron mobility, and $\tilde{\epsilon}$ is the low-frequency dielectric constant.

Equation (1) expresses the generation rate of ionized donors and electrons that is due to the interaction with the optical field, Eq. (2) is the Gauss law, Eq. (3) is the continuity equation, and charge and current densities are expressed by Eqs. (4) and (5), respectively.

If we apply a static voltage $V$ across the crystal, orthogonally to the beam propagation direction (which we take to be the $z$ axis; see Fig. 1), the space-charge field must also satisfy the following integral constraint:

$$
V=-\int_{-l / 2}^{l / 2} \mathbf{E} \cdot \mathrm{d} \mathbf{s},
$$

where $l$ is the distance between the two crystal faces to which the external bias voltage is applied. The set of Eqs. (1)-(5) has to be supplemented by an equation that describes the evolution of the monochromatic optical field $\mathbf{E}_{\text {opt }}$ propagating in the PR medium, whose intensity $I$ appears in Eq. (1). In the standard paraxial approximation that equation reads as

$$
\left(i \frac{\partial}{\partial z}+\frac{1}{2 k} \nabla_{\perp}^{2}\right) \mathbf{A}+\frac{k}{n_{1}} \Delta \mathbf{n}: \mathbf{A}=0,
$$

where we set $\mathbf{E}_{\text {opt }}(x, y, z, t)=\mathbf{A}(x, y, z) \exp (i k z-i \omega t)$ and $\nabla_{\perp}=(\partial / \partial x, \partial / \partial y) ; k=(\omega / c) n_{1}$ and $n_{1}$ are the optical wave vector and the linear (unperturbed) refractive index of the crystal, respectively; and $\Delta \mathbf{n}$ is the tensorial refractive-index variation associated with the linear electro-optics (i.e., Pockels) tensor r:

$$
\Delta \mathbf{n}=-\frac{n_{1}^{3}}{2} \mathbf{r}: \mathbf{E} .
$$

Equations (1)-(8) are the general system of coupled material and wave equations that model light propagation in a PR crystal.

We actually look for a steady-state solution (i.e., $\partial / \partial t$ $=0$ ), and our first task is to obtain from Eqs. (1)-(5) a single equation for $\mathbf{E}$ that does not contain the other two dependent variables $N_{D}{ }^{+}$and $N_{e}$. We accomplish this in one of two ways, according to whether we are dealing with a $1+1-\mathrm{D}$ or a $2+1-\mathrm{D}$ case. More precisely, because we will be looking for self-trapped solutions, we can assume that the optical intensity $I=|\mathbf{A}|^{2}$ is a function of $x$ alone $(1+1$-D case $)$ or of $(x, y)$ alone $(2+1$-D case $)$, accordingly, and set $\mathbf{E}=\mathbf{E}(x)$ or $\mathbf{E}=\mathbf{E}(x, y)$, respectively.
Besides, in the $1+1$-D case, if the bias field is applied along the $x$ axis we can look for a scalar field $\mathbf{E}=E(x) \hat{e}_{x}$ aligned along the $x$ axis.

Moreover, in both cases the space-charge field $\mathbf{E}$ and the electron density $N_{e}$ are expected to take constant boundary values, say,

$$
\mathbf{E}=E_{\infty} \hat{e}_{x}, \quad N_{e}=N_{e \infty},
$$

on the crystal surfaces $x= \pm l / 2$ between which voltage $V$ is applied.

\section{1 + 1-DIMENSIONAL CASE}

In the $1+1$-D case the continuity equation can be integrated to give

$$
\frac{\mathrm{d} N_{e}}{\mathrm{~d} x}+\frac{q}{K_{B} T}\left(E N_{e}-E_{\infty} N_{e \infty}\right)=0,
$$

where we have explicitly related the integration constant to the boundary values $E_{\infty}=E( \pm l / 2)$ of the space-charge field and $N_{e^{\infty}}=N_{e}( \pm l / 2)$ of the electron density on the crystal faces where the bias field in applied [see Eq. (9)]. This relation follows from the assumption that the deviation from $E_{\infty}$ and $N_{e^{\infty}}$ that is due to the light beam is well localized, namely, that the derivative of $E(x)$ and $N_{e}(x)$ vanishes at the crystal faces, i.e., $\mathrm{d} E( \pm l / 2) / \mathrm{d} x$ $=\mathrm{d} N_{e}( \pm l / 2) / \mathrm{d} x=0 . \quad E_{\infty}$ and $N_{e \infty}$ obviously depend on the specified asymptotic behavior of $I(x)$ [i.e., $I( \pm l / 2)$ $\sim 0$ or $I( \pm l / 2)=I_{\infty} \neq 0$, according to whether one is dealing with bright or dark solitons, respectively], and their value is determined only after our boundary-value problem, with integral condition (6), is solved. The dependent variable $N_{D}{ }^{+}(x)$ is easily expressed in terms of $N_{e}(x)$ by use of balance equation (1), whereas, from Eqs. (1), (2), and (4) and the (well-justified) assumption that $N_{A} \gg N_{e}$, it follows that

$$
N_{e}=\frac{(q / \tilde{\boldsymbol{\epsilon}})\left(N_{D}-N_{A}\right)-E^{\prime}}{(q / \tilde{\boldsymbol{\epsilon}}) N_{A}+E^{\prime}}\left(s_{1} I+\beta_{1}\right),
$$

where $s_{1}=s / \gamma, \beta_{1}=\beta / \gamma$, and the prime stands for a derivative with respect to $x$. The introduction of Eq. (11) into Eq. (10) allows us to obtain a single equation for $E(x)$. More precisely, by setting $\alpha=\left(N_{D}-N_{A}\right) / N_{A}$ and by introducing the natural dimensionless variables

$$
Y \equiv \frac{E}{E_{D}}, \quad \xi \equiv k_{D} x, \quad u \equiv \frac{A}{\sqrt{I_{d}}}, \quad \bar{N} \equiv \frac{N_{e}}{\beta_{1}}
$$

together with the definitions

$$
\begin{aligned}
k_{D}{ }^{2} & \equiv \frac{q^{2} N_{A}}{\tilde{\epsilon} K_{B} T}, \quad E_{D} \equiv \frac{K_{B} T}{q} k_{D}, \quad I_{d} \equiv \frac{\beta_{1}}{s_{1}}, \\
Y_{\infty} & \equiv \frac{E_{\infty}}{E_{D}}, \quad \bar{N}_{\infty} \equiv \frac{N_{e^{\infty}}}{\beta_{1}},
\end{aligned}
$$

we obtain 


$$
\begin{gathered}
\frac{\mathrm{d}^{2} Y}{\mathrm{~d} \xi^{2}}-\frac{N_{A}}{N_{D}}\left(\alpha-\frac{\mathrm{d} Y}{\mathrm{~d} \xi}\right)\left(1+\frac{\mathrm{d} Y}{\mathrm{~d} \xi}\right)\left[\frac{\mathrm{d}}{\mathrm{d} \xi} \ln \left(1+|u|^{2}\right)+Y\right] \\
+\frac{N_{A} \bar{N}_{\infty}}{N_{D}} Y_{\infty}\left(1+\frac{\mathrm{d} Y}{\mathrm{~d} \xi}\right)^{2} \frac{1}{1+|u|^{2}}=0
\end{gathered}
$$

If we assume that the derivatives of $Y$ are negligible (this assumption has to be justified a posteriori), Eq. (14) becomes linear and yields the approximate solution

$$
Y=\frac{1}{\alpha} \bar{N}_{\infty} \frac{Y_{\infty}}{1+|u|^{2}}-\frac{\mathrm{d}}{\mathrm{d} \xi} \ln \left(1+|u|^{2}\right) .
$$

Because of our boundary conditions, $\bar{N}_{\infty}$ is easily obtained from Eq. (11), which yields

$$
\bar{N}_{\infty}=\alpha\left(1+\left|u_{\infty}\right|^{2}\right),
$$

where $\left|u_{\infty}\right|^{2} \equiv I( \pm l / 2) / I_{d}$, so $\left|u_{\infty}\right|^{2}=0$ for bright solitons and $\left|u_{\infty}\right|^{2}=I_{\infty} / I_{d}$ for dark solitons. To evaluate $Y_{\infty}$ we have to insert Eq. (15) into Eq. (6). In this way we obtain, in the significant case of $l_{s} \ll l$,

$$
Y_{\infty}=-\frac{1}{E_{D}} \frac{V}{l} .
$$

By inserting Eqs. (16) and (17) into Eq. (15) and going back to dimensional units for the space-charge field we finally obtain

$$
\begin{aligned}
E= & -\frac{V}{l} \frac{1}{1+|u|^{2}} \\
& -\frac{E_{D}}{k_{D}} \frac{\mathrm{d}}{\mathrm{d} x} \ln \left(1+|u|^{2}\right) \quad \text { (bright solitons), } \\
E= & -\frac{V}{l} \frac{1+\left|u_{\infty}\right|^{2}}{1+|u|^{2}} \\
& -\frac{E_{D}}{k_{D}} \frac{\mathrm{d}}{\mathrm{d} x} \ln \left(1+|u|^{2}\right) \quad \text { (dark solitons). }
\end{aligned}
$$

In the above derivation leading to the approximate expressions Eqs. (18) and (19), we have assumed that $|u|^{2}$ differs from its boundary value $\left|u_{\infty}\right|^{2}$ only in a small interval of size $l_{s}$. Actually, $|u|^{2}$ has to be determined in a self-consistent way by solution of wave equation (7), which describes propagation in the PR crystal, where $A(x, z)$ varies as a function of $z$ on a scale much longer than $1 / k$. As we are looking for self-trapped solutions, we set $A(x, z)=\exp (i \delta z) A(x)$, and parabolic wave equation (7) reduces to the following ordinary differential equation for the normalized amplitude $u(x)=A(x) / \sqrt{I_{d}}$ :

$$
\frac{1}{2 k} \frac{\mathrm{d}^{2}}{\mathrm{~d} x^{2}} u=\delta u-\frac{k}{n_{1}} \Delta n u,
$$

where, in the scalar approximation, the refractive-index variation is $\Delta n=-1 / 2 n_{1}{ }^{3} \hat{r} E$, where $\hat{r}$ is the tensor element that is most effective in the adopted geometry. Equations (18)-(20) are a set of nonlinear coupled equations that describe propagation of a beam that is selftrapped in one transverse dimension in a PR crystal. They have been extensively investigated in Refs. 24, 25, and 27 , where bright and dark solitary-wave solutions in one transverse dimension were shown to exist, and $E_{\infty}$ was evaluated analytically for the different cases of bright and dark solitons. ${ }^{27}$ The PR effect, which has an intrinsically nonlocal nature in space, under the approximation that leads to Eq. (17) is accounted for in local terms by a refractive-index variation $\Delta n$, which becomes, whenever $l_{s} \ll l$ and if the derivative terms on the right-hand sides of Eqs. (18) and (19) are omitted (for $l_{D} \ll l_{s} \ll l$ these terms lead to self-bending of the soliton beam, as predicted in Ref. 39 and as observed in Ref. 30), of the saturated Kerr type ${ }^{9}$; that is,

$$
\Delta n \propto \frac{1}{1+|u|^{2}} .
$$

In this case, for bright solitons the nonlinear wave equation takes the form

$$
i \frac{\partial u}{\partial \hat{\zeta}}+\frac{\partial^{2} u}{\partial \hat{\xi}^{2}}-s \frac{u}{1+|u|^{2}}=0,
$$

and for dark solitons it is

$$
i \frac{\partial u}{\partial \hat{\zeta}}+\frac{\partial^{2} u}{\partial \hat{\xi}^{2}}-s \frac{1+\left|u_{\infty}\right|^{2}}{1+|u|^{2}} u=0,
$$

where we have introduced new variables expressed in terms of a dimensionless parameter $b$ as

$$
\begin{aligned}
& \hat{\xi} \equiv \sqrt{b} \xi=k n_{1}\left(\frac{|\hat{r} V|}{l}\right)^{1 / 2} x, \\
& \hat{\zeta} \equiv \frac{k_{D}{ }^{2}}{2 k} b z=\frac{k n_{1}{ }^{2}}{2} E_{D}\left|\hat{r} Y_{\infty}\right| z, \\
& b=\frac{k^{2} n_{1}{ }^{2}}{k_{D}{ }^{2}} E_{D}\left|\hat{r} Y_{\infty}\right|,
\end{aligned}
$$

and $s= \pm 1$, depending on the relative orientation of the bias electric field with respect to the $c$ axis of the crystal, namely, $s \equiv \operatorname{sign}\left(\hat{r} Y_{\infty}\right)$. The soliton's existence [which corresponds to $s=1$ in Eq. (22) and to $s=-1$ in Eq. (23)] turns out to depend in a unique way on the values of its width, peak amplitude $|u(0)|$ (bright) and boundary amplitude $\left|u_{\infty}\right|$ (dark), and bias field $V / l$. These existence curves are shown in Fig. 2, where the bright (dark) soliton diameter in normalized units $\hat{\xi}$ is plotted as a

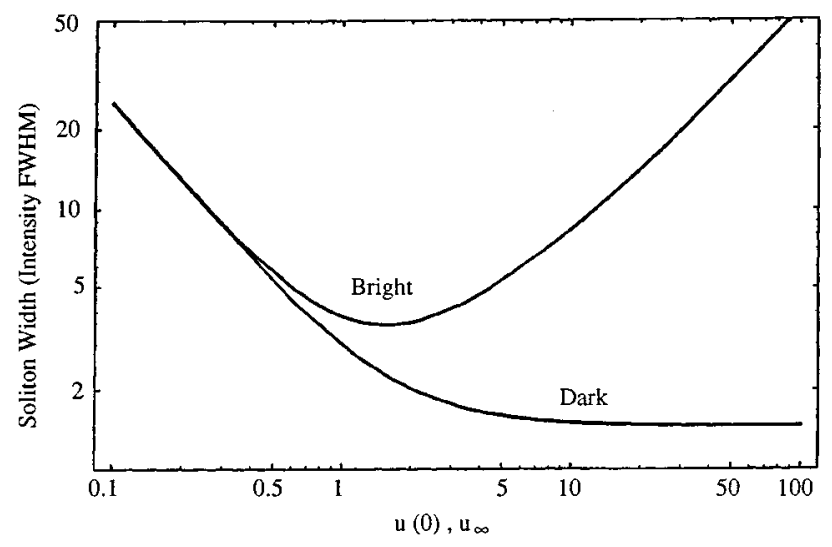

Fig. 2. Existence curves for $1+1$-D solitons. 
function of $|u(0)|\left(\left|u_{\infty}\right|\right)$. An important attribute of the soliton existence curve is that it is a single-valued function, which implies that for a given crystal a soliton of a specific diameter at a given value of the intensity ratio exists only at a single value of the external field. ${ }^{24,27,33,35,43}$

\section{2 + 1-DIMENSIONAL CASE}

Here we show that new features of the model [Eqs. (1)(5), (7), and (8)] arise because of higher dimensionality. In the general case one should specify the form of the electro-optic tensor that appears in Eq. (8) and is to be used in Eq. (7). The general tensorial problem is rather involved and leads to coupled equations for the polarization components of the optical field, but we do not address this problem here (for a discussion of $1+1$-D PR vector solitons see Ref. 38). However, in practice, by suitably choosing the experimental geometry one can find a crystalline configuration that does not rotate the polarization and which has one electro-optic coefficient much larger than the others (for example, strontium barium niobate $^{24,27,30,31}$ ) in such a way that an optical field initially polarized along the $x$ axis maintains its linear polarization; then the problem can be adequately formulated as a scalar one.

The set of Eqs. (1)-(5) and (7) can be reduced to a system of three equations after $N_{D}{ }^{+}$is eliminated. More precisely, Eqs. (2) and (3) yield, respectively,

$$
\begin{aligned}
\tilde{\nabla}^{2} \phi & =\frac{\bar{N}-\alpha\left(1+|u|^{2}\right)}{\bar{N}+1+|u|^{2}}, \\
\tilde{\nabla}^{2} \bar{N} & =\tilde{\nabla} \cdot(\bar{N} \tilde{\nabla} \phi),
\end{aligned}
$$

whereas wave equation (7) becomes $\left[s \equiv \operatorname{sign}\left(\hat{r} Y_{\infty}\right)\right]$

$$
\left(i 2 \frac{k}{k_{D}} \frac{\partial}{\partial \zeta}+\tilde{\nabla}_{\perp}^{2}\right) u=-s \frac{b}{Y_{\infty}} \frac{\partial \phi}{\partial \xi} u, \quad s= \pm 1 .
$$

In Eqs. (25)-(27) we have introduced the potential $\phi(\xi, \eta, \zeta)$ associated with the static field $\mathbf{Y}=\mathbf{E} / E_{D}$, namely,

$$
\mathbf{Y}=-\tilde{\nabla} \phi
$$

and we have adopted the dimensionless variables introduced in Section 3 [see Eqs. (12) and (13)], together with the additional notation

$$
\begin{aligned}
& \eta \equiv k_{D} y, \quad \zeta \equiv k_{D} z, \\
& \tilde{\nabla}=(\partial / \partial \xi, \partial / \partial \eta, \partial / \partial \zeta), \quad \widetilde{\nabla}_{\perp}=(\partial / \partial \xi, \partial / \partial \eta) .
\end{aligned}
$$

By assuming hereafter that the bias field is generated by voltage $V$ applied to the crystal faces orthogonal to the $x$ axis, we observe that in the trivial case in which the intensity is constant in the whole crystal, $|u|^{2}=\left|u_{\infty}\right|^{2}$, the basic Eqs. (27), (25), and (26), together with boundary condition (6) (and neglecting fringe effects at the crystal surface), have the simple solution

$$
\begin{aligned}
& \phi=\frac{V}{l E_{D}} \xi, \quad \bar{N}=\bar{N}_{\infty}=\alpha\left(1+\left|u_{\infty}\right|^{2}\right), \\
& u=\left|u_{\infty}\right| \exp \left(-\frac{i k_{D}}{2 k} s b \zeta\right),
\end{aligned}
$$

which corresponds to an optical field plane wave whose phase velocity is $c /\left[n_{1}\left(1+n_{1}^{2} \hat{r} V / 2 l\right)\right]$. Therefore, whenever the intensity $|u|^{2}$ behaves like a constant $\left|u_{\infty}\right|^{2}$ in proximity to the crystal transverse boundaries, it is expedient to introduce the new potential

$$
\psi(\hat{\xi}, \hat{\eta}, \hat{\zeta})=-\hat{\xi}-\frac{\sqrt{b}}{Y_{\infty}}\left(\phi-\ln \frac{\bar{N}}{\bar{N}_{\infty}}\right)
$$

as a function of the rescaled variables

$$
(\hat{\xi}, \hat{\eta}) \equiv \sqrt{b}(\xi, \eta)
$$

together with the rescaled distance $\hat{\zeta}$ introduced in Eqs. (24). Moreover, when we conveniently change the phase of the amplitude $u$ through the transformation

$$
\hat{u}(\hat{\xi}, \hat{\eta}, \hat{\zeta})=u \exp (i s \hat{\zeta})
$$

propagation equation (27) takes the form

$$
\left(i \frac{\partial}{\partial \hat{\zeta}}+\hat{\nabla}_{\perp}^{2}\right) \hat{u}=s\left(\frac{\partial \psi}{\partial \hat{\xi}}-\frac{\sqrt{b}}{Y_{\infty}} \frac{\partial}{\partial \hat{\xi}} \ln \bar{N}\right) \hat{u}, \quad s= \pm 1,
$$

where $\hat{\nabla}_{\perp}=(\partial / \partial \hat{\xi}, \partial / \partial \hat{\eta})$. We obtain a simpler form of Eq. (27) by dropping the derivative term on its right-hand side on the basis that this term merely accounts for a transverse deflection of the beam, ${ }^{48}$ namely,

$$
\left(i \frac{\partial}{\partial \hat{\zeta}}+\hat{\nabla}_{\perp}^{2}\right) \hat{u}=s\left(\frac{\partial \psi}{\partial \hat{\xi}}\right) \hat{u}, \quad s= \pm 1 .
$$

To solve Eq. (36) we have to derive the function $\psi$ by in tegrating the other two elliptical equations (25) and (26), rewritten in the new variables, with the boundary conditions

$$
\psi\left( \pm \sqrt{b} k_{D} \frac{l}{2}, \hat{\eta}, \hat{\zeta}\right)=\psi\left(\hat{\xi}, \pm \sqrt{b} k_{D} \frac{l}{2}, \hat{\zeta}\right)=0 .
$$

In this respect we note that the role of the linear term on the right-hand side of definition (32) is to move to infinity the vanishing boundary condition (37) whenever the transverse dimensions of the beam are much smaller than the crystal transverse dimension $l$; thus hereafter we adopt the vanishing asymptotic condition

$$
\psi(\hat{\xi}, \hat{\eta}, \hat{\zeta}) \rightarrow 0 \quad \text { as } \quad \hat{\xi}^{2}+\hat{\eta}^{2} \rightarrow \infty .
$$

In what follows, we do not approach the problem of the evolution in the $\hat{\zeta}$ variable of an input pulse $\hat{u}(\hat{\xi}, \hat{\eta}, \hat{\zeta}$ $=0$ ). Instead, we focus our attention on the stationary trapped (soliton) solutions of the form

$$
\hat{u}(\hat{\xi}, \hat{\eta}, \hat{\zeta})=W(\hat{\xi}, \hat{\eta}) \exp (i \gamma \hat{\zeta})
$$

where the amplitude $W$ is defined on the transverse plane $(\hat{\xi}, \hat{\eta})$, and $\gamma$, the propagation constant, is a real parameter. Equation (39) simplifies not only propagation equation (36), which now reads as 


$$
\hat{\nabla}_{\perp}^{2} W=\left(\gamma+s \frac{\partial \psi}{\partial \hat{\xi}}\right) W, \quad s= \pm 1,
$$

but also the other two equations (25) and (26), because we can assume, as well, that the function $\psi$ and the (rescaled) electron density $\bar{N}$ are independent of the longitudinal variable $\hat{\zeta}$. Therefore, by taking into account definition (32), Eq. (26) shows that $\psi$ has to satisfy the relation

$$
\hat{\nabla}_{\perp} \cdot\left(\bar{N} \hat{\nabla}_{\perp} \psi\right)=-\frac{\partial}{\partial \hat{\xi}} \bar{N}, \quad \bar{N}=\bar{N}(\hat{\xi}, \hat{\eta}),
$$

and the electric potential $\phi=\phi(\hat{\xi}, \hat{\eta})$ obeys Eq. (25) in its rescaled form:

$$
\hat{\nabla}_{\perp}^{2} \phi=\frac{1}{b} \frac{\bar{N}-\alpha\left(1+|W|^{2}\right)}{\bar{N}+1+|W|^{2}} .
$$

A soliton solution of these planar coupled equations, if it exists, is characterized by the following asymptotic conditions (whenever convenient, we use polar coordinates $\hat{\xi}$ $=\rho \cos \theta, \hat{\eta}=\rho \sin \theta)$ : Both the beam intensity and the electron density have to reach a constant value at infinity, i.e.,

$$
|W| \rightarrow\left|W_{\infty}\right| \quad \text { as } \quad \rho \rightarrow \infty,
$$

where $\left|W_{\infty}\right|=0$ for bright solitons and $\left|W_{\infty}\right| \neq 0$ for dark solitons, whereas

$$
\bar{N} \rightarrow \alpha\left(1+\left|W_{\infty}\right|^{2}\right)=\bar{N}_{\infty} \quad \text { as } \quad \rho \rightarrow \infty .
$$

Because proving the existence of soliton solutions is a difficult task, we content ourselves with a consistency proof. In fact, in Appendix A we show that the asymptotic behavior [relation (44)] implies that $\psi$ vanishes as $\rho \rightarrow \infty$ as $O(1 / \rho)$, and therefore [see Eq. (32)] that

$$
\phi+Y_{\infty} \xi \rightarrow 0 \text { as } \rho \rightarrow \infty,
$$

consistent with Eq. (42). Let us now turn our attention to the phase $\varphi(\hat{\xi}, \hat{\eta})$ of the soliton, namely, $W$ $=|W| \exp (i \varphi)$. For bright solitons we first observe that an obvious necessary condition for their existence is that the propagation constant $\gamma$ in Eq. (40) be positive, $\gamma$ $>0$. Second, as shown in Appendix A, the asymptotic vanishing of the amplitude, $W_{\infty}=0$ [see relation (43)], implies that the soliton phase is necessarily constant, i.e., that

$$
\hat{\nabla}_{\perp} \varphi=0 .
$$

At the same time, for dark solitons, namely, $\left|W_{\infty}\right| \neq 0$, combining Eq. (A7) below with relation (38) shows that the parameter $\gamma$ in Eq. (40) cannot be positive, $\gamma=-p^{2}$ $\leqslant 0$, and the asymptotic phase $\varphi_{\infty}(\hat{\xi}, \hat{\eta})$, say,

$$
\varphi \rightarrow \varphi_{\infty} \text { as } \rho \rightarrow \infty,
$$

must have the following expression (see Appendix A):

$$
\varphi_{\infty}=p(\hat{\xi} \sin \delta+\hat{\eta} \cos \delta)+m \theta,
$$

where $\delta$ is a constant angle and $m$ is an integer. However, if the dark soliton's amplitude $|W|$ is $\theta$ independent (see below), then (Appendix A) the soliton propagation constant $\gamma$ must be identically zero, namely, $p=0$ and $\varphi_{\infty}=m \theta$. Indeed, in this case the soliton phase itself takes the simple expression $\varphi=m \theta$ (Appendix A), which is consistent with that associated with dark vortex solitons ${ }^{22,51-53}$; the integer $m$ represents the topological charge. The condition $\gamma=0$ also implies that $\hat{u}(\hat{\xi}, \hat{\eta}, \hat{\zeta})=W(\hat{\xi}, \hat{\eta})$, that is, a stationary solution in $\hat{\zeta}$. Recalling the definition of $\hat{\zeta}$, we find that this means that the propagation constant of the $2+1$-D dark vortex soliton will be identical to the propagation constant of a plane wave propagating along the same crystal and biased by the same field. Furthermore, this also means that all dark vortex solitons propagate at the same phase velocity, regardless of their plateau amplitude $W_{\infty}$. This property is in contrast to that for bright solitons, whose propagation constant $\gamma$ depends on their peak amplitude as described below. It is interesting that a similar situation occurs for $1+1$-D solitons, which propagate with the same phase velocity as a plane wave does in the same medium biased by the same field, independently of the soliton peak amplitude $W(0) .^{27}$ The propagation constants of $1+1-\mathrm{D}$ and $2+1-\mathrm{D}$ dark solitons are, therefore, identical.

\section{SELF-TRAPPED BEAM PROPAGATION}

To treat the nonlinear $2+1-\mathrm{D}$ propagation problem more specifically we have to look for an expression of the normalized space-charge field $\mathbf{Y}=\mathbf{E} / E_{D}$ in terms of the optical intensity. To this end, we start by eliminating $\mathrm{N}_{D}{ }^{+}$between Eq. (1) (written in the stationary case, where $\partial / \partial t=0)$ and Eq. (2), thus getting, with the help of Eq. (4),

$$
\bar{N}=\frac{\alpha-\tilde{\nabla} \cdot \mathbf{Y}}{\mathbf{1}+\tilde{\nabla} \cdot \mathbf{Y}} Q,
$$

where we have assumed that $N_{e} \ll N_{A}$ and have introduced

$$
Q=1+|u|^{2} \text {. }
$$

Equation (49) can now be inserted into the stationary version of Eq. (3), which yields, after we take advantage of Eq. (5),

$$
\tilde{\nabla} \cdot\left[\frac{\alpha-\tilde{\nabla} \cdot \mathbf{Y}}{1+\tilde{\nabla} \cdot \mathbf{Y}} Q \mathbf{Y}+\tilde{\nabla}\left(\frac{\alpha-\tilde{\nabla} \cdot \mathbf{Y}}{1+\tilde{\nabla} \cdot \mathbf{Y}} \boldsymbol{Q}\right)\right]=0 .
$$

Equation (51) can be cast in a simpler approximate form if we can neglect $\tilde{\nabla} \cdot \mathbf{Y}$ with respect to 1 (and thus with respect to $\alpha$, which is typically much larger than 1 ), a hypothesis that has to be self-consistently checked $a$ posteriori once $\mathbf{Y}$ has been worked out. Under this assumption, Eq. (51) becomes

$$
\tilde{\nabla} \cdot(Q \mathbf{Y}+\tilde{\nabla} \boldsymbol{Q})=0 .
$$

We remark that one could think at this point that (apart from the diffusion term) Eq. (52) is simply the continuity equation $\nabla \cdot(\sigma \mathbf{E})=0$, once the conductivity $\sigma$ is assumed to be proportional to the total optical intensity, ${ }^{54}$ and that, consequently, most of the previous theoretical effort could have been spared. Actually, Eq. (52) provides the theoretical justification, to the lowest significant 
order in $\tilde{\nabla} \cdot \mathbf{Y}$ only, of the validity of such an equation, which, because it is phenomenological, cannot be taken for granted a priori.

Next we consider situations (self-trapped beams) in which the light intensity $I$ is independent of $z$ (or, at least, in which its scale of variation over $z$ is much larger than those over $x$ and $y$ ), so that, at least when we are solving Eq. (52), $\mathbf{Y}$ and $Q$ can be assumed to depend only on the transverse coordinates $x$ and $y$.

The most general solution of Eq. (52), together with the proper boundary conditions $E_{y}=E_{z}=0$ for $x= \pm l / 2$ and $E_{x}(x=l / 2)=E_{x}(x=-l / 2)=E_{D} Y_{\infty}$, can be written in the form

$$
\begin{aligned}
\mathbf{Y}(\xi, \eta)= & \frac{\tilde{Y}_{\infty}}{1+|u|^{2}} \hat{e}_{x}-\tilde{\nabla}_{\perp} \ln \left(1+|u|^{2}\right) \\
& +\frac{\tilde{Y}_{\infty}}{1+|u|^{2}}\left(\hat{e}_{x} \frac{\partial}{\partial \eta} f-\hat{e}_{y} \frac{\partial}{\partial \xi} f\right),
\end{aligned}
$$

where $\tilde{Y}_{\infty}=Y_{\infty}\left(1+\left|u_{\infty}\right|^{2}\right) ; \hat{e}_{x}$ and $\hat{e}_{y}$ are unit vectors in the $x$ and the $y$ directions, respectively; and $f(\xi, \eta)$ is an arbitrary function that we can determine by imposing the condition that

$$
\tilde{\nabla}_{\perp} \times \mathbf{Y}=0 .
$$

By doing that we obtain an equation for $f(\xi, \eta)$ that reads as

$$
\tilde{\nabla}_{\perp}^{2} f-\tilde{\nabla}_{\perp} f \cdot \tilde{\nabla}_{\perp} \ln Q=\frac{\partial}{\partial \eta} \ln Q .
$$

Inasmuch as a general analytical solution of Eq. (55) is not available, we first consider below the limiting case in which $|u(0)|^{2},\left|u_{\infty}\right|^{2} \ll 1$.

In this situation the solution of Eq. (55) can easily be worked out if we neglect (an assumption that has to be justified a posteriori) the second term on its left-hand side. Because $f(\xi, \eta)$ has to vanish at infinity, the solution can be expressed in terms of the two-dimensional Green function as

$$
\begin{aligned}
f(\xi, \eta)= & \frac{1}{2 \pi} \iint \mathrm{d} \xi^{\prime} \mathrm{d} \eta^{\prime} \ln \left\{\left[\left(\xi-\xi^{\prime}\right)^{2}\right.\right. \\
& \left.\left.+\left(\eta-\eta^{\prime}\right)^{2}\right]^{1 / 2}\right\} \frac{\partial}{\partial \eta^{\prime}} \ln Q\left(\xi^{\prime}, \eta^{\prime}\right) .
\end{aligned}
$$

We can now evaluate

$$
\begin{aligned}
\frac{\partial}{\partial \eta} f(\xi, \eta)= & \frac{1}{2 \pi} \iint \mathrm{d} \xi^{\prime} \mathrm{d} \eta^{\prime} \frac{\eta-\eta^{\prime}}{\left(\xi-\xi^{\prime}\right)^{2}+\left(\eta-\eta^{\prime}\right)^{2}} \\
& \times \frac{\partial}{\partial \eta^{\prime}} \ln Q\left(\xi^{\prime}, \eta^{\prime}\right), \\
-\frac{\partial}{\partial \xi} f(\xi, \eta)= & \frac{1}{2 \pi} \iint \mathrm{d} \xi^{\prime} \mathrm{d} \eta^{\prime} \frac{\xi^{\prime}-\xi}{\left(\xi-\xi^{\prime}\right)^{2}+\left(\eta-\eta^{\prime}\right)^{2}} \\
& \times \frac{\partial}{\partial \eta^{\prime}} \ln Q\left(\xi^{\prime}, \eta^{\prime}\right),
\end{aligned}
$$

which, after we introduce polar coordinates according to the relations

$$
\rho \cos \theta=\xi^{\prime}-\xi, \quad \rho \sin \theta=\eta^{\prime}-\eta,
$$

can be rewritten as

$$
\begin{aligned}
\frac{\partial}{\partial \eta} f(\xi, \eta)= & -\frac{1}{2 \pi} \iint \mathrm{d} \rho \mathrm{d} \theta \\
& \times \sin \theta\left(\sin \theta \frac{\partial}{\partial \rho}+\frac{\cos \theta}{\rho} \frac{\partial}{\partial \theta}\right) \\
& \times \ln Q(\xi+\rho \cos \theta, \eta+\rho \sin \theta), \\
-\frac{\partial}{\partial \xi} f(\xi, \eta)= & \frac{1}{2 \pi} \iint \mathrm{d} \rho \mathrm{d} \theta \\
& \times \cos \theta\left(\sin \theta \frac{\partial}{\partial \rho}+\frac{\cos \theta}{\rho} \frac{\partial}{\partial \theta}\right) \\
& \times \ln Q(\xi+\rho \cos \theta, \eta+\rho \sin \theta) .
\end{aligned}
$$

We can now split the right-hand-side integrals of Eqs. (60) and (61) into local and nonlocal contributions by integrating by parts and using the pertinent boundary conditions. In this way we get

$$
\begin{aligned}
\frac{\partial}{\partial \eta} f(\xi, \eta)= & \frac{1}{2} \ln \frac{1+|u|^{2}}{1+\left|u_{\infty}\right|^{2}}+\frac{\tilde{Y}_{\infty}}{2 \pi} \iint \frac{\mathrm{d} \rho \mathrm{d} \theta}{\rho} \cos (2 \theta) \\
& \times \ln Q(\xi+\rho \cos \theta, \eta+\rho \sin \theta) \\
-\frac{\partial}{\partial \xi} f(\xi, \eta)= & \frac{1}{2 \pi} \iint \frac{\mathrm{d} \rho \mathrm{d} \theta}{\rho} \sin (2 \theta) \\
& \times \ln Q(\xi+\rho \cos \theta, \eta+\rho \sin \theta)
\end{aligned}
$$

so, by omitting the diffusion term $\widetilde{\nabla}_{\perp} \ln \left(1+|u|^{2}\right)$ in Eq. (53), we finally obtain for the space-charge field $\mathbf{Y}$ the expression

$$
\begin{aligned}
\mathbf{Y}= & Y_{\infty} \frac{\left(1+\left|u_{\infty}\right|^{2}\right)}{1+|u|^{2}}\left(1+\frac{1}{2} \ln \frac{1+|u|^{2}}{1+\left|u_{\infty}\right|^{2}}\right) \hat{e}_{x} \\
& +\frac{Y_{\infty}}{2 \pi} \frac{1+\left|u_{\infty}\right|^{2}}{1+|u|^{2}} \iint \frac{\mathrm{d} \rho \mathrm{d} \theta}{\rho} \cos (2 \theta) \\
& \times \ln \left[1+|u(\xi+\rho \cos \theta, \eta+\rho \sin \theta)|^{2}\right] \hat{e}_{x} \\
& +\frac{Y_{\infty}}{2 \pi} \frac{1+\left|u_{\infty}\right|^{2}}{1+|u|^{2}} \iint \frac{\mathrm{d} \rho \mathrm{d} \theta}{\rho} \sin (2 \theta) \\
& \times \ln \left[1+|u(\xi+\rho \cos \theta, \eta+\rho \sin \theta)|^{2}\right] \hat{e}_{y} .
\end{aligned}
$$

Note that, if we keep only the local contribution proportional to $1 /\left(1+|u|^{2}\right)$, the one- and two-dimensional expressions for the space-charge field have the same form.

At this point we observe that, unlike for the $1+1-\mathrm{D}$ case, the condition given in Eq. (6) yields quite generally Eq. (17), because the path can be chosen in the $(x, y)$ plane in a region in which the illumination is uniform.

We are now in a position to check in a self-consistent way the validity of the two approximations that consisted in assuming $\widetilde{\nabla}_{\perp} \cdot \mathbf{Y}$ small with respect to 1 and in neglecting the second term on the left-hand side of Eq. (55) and to verify that both approximations are fulfilled in the limit $|u|^{2} \ll 1$. 
In the previous formula [Eq. (64)], $|u|^{2}$ appeared merely as a given function. In fact, however, the amplitude $u$ depends on $\mathbf{Y}$ through the parabolic wave equation for the propagating optical (high-frequency) field, as explicitly shown by Eqs. (27) and (28), which are equivalent to

$$
\left(i \frac{\partial}{\partial \hat{\zeta}}+\hat{\nabla}_{\perp}^{2}\right) u=s \frac{Y_{x}}{Y_{\infty}} u .
$$

Indeed, in our approximation scheme this propagation equation, together with Eq. (64), takes the following form of an integrodifferential equation:

$$
\begin{aligned}
\left(i \frac{\partial}{\partial \hat{\zeta}}+\hat{\nabla}_{\perp}^{2}\right) u= & s \frac{\left(1+\left|u_{\infty}\right|^{2}\right)}{1+|u|^{2}}\left(1+\frac{1}{2} \ln \frac{1+|u|^{2}}{1+\left|u_{\infty}\right|^{2}}\right) u \\
& +\frac{s}{2 \pi} \frac{1+\left|u_{\infty}\right|^{2}}{1+|u|^{2}} u \iint \frac{\mathrm{d} \rho \mathrm{d} \theta}{\rho} \cos (2 \theta) \\
& \times \ln [1+\mid u(\hat{\xi}+\rho \cos \theta, \hat{\eta} \\
& \left.+\rho \sin \theta)\left.\right|^{2}\right] .
\end{aligned}
$$

Note that we have always omitted on the right-hand side of propagation equation (66) a linear term $-(\alpha / 2) u$ that accounts for the losses of the medium (which can be significant; $\alpha \cong 1 \mathrm{~cm}^{-1}$ ). We can justify doing this by observing that the dark irradiance $I_{d}$, which is associated with thermal charge-carrier generation, is artificially increased in screening-soliton experiments ${ }^{29,30}$ by illumination of the whole crystal with a background uniform light of intensity $I_{b}$ (artificial irradiance), so $u=A /\left(I_{d}\right.$ $\left.+I_{b}\right)^{1 / 2}$. If $I_{d} \ll I_{b}$, and if $I_{b}$ undergoes the same losses as $A$, then the form of Eq. (66) ensures that the loss term can be omitted because $u=A / I_{b}{ }^{1 / 2}=A \exp (-\alpha z / 2)$ / $I_{b}{ }^{1 / 2} \exp (-\alpha z / 2)$. This is also true for $|u| \ll 1$, when the PR nonlinearity becomes of the Kerr type, whereas in a standard Kerr nonlinearity the soliton attenuates at twice the rate of a linear signal. ${ }^{55}$

By inspection of the right-hand side of Eq. (66) it is apparent that the nonlocal contribution is asymmetric and that this circumstance prevents the existence of transversely symmetric solutions. However, in the regime of $|u(0)|^{2},\left|u_{\infty}\right|^{2} \ll 1$, where this term is small with respect to the symmetric terms, one can look for self-trapped symmetric solutions (spatial solitons) of the equation obtained by dropping the term (local approximation).

To carry out this program we introduce the function $W(\hat{\xi}, \hat{\eta})$, defined by

$$
u(\hat{\xi}, \hat{\eta}, \hat{\zeta})=W(\hat{\xi}, \hat{\eta}) \exp (i \beta \hat{\zeta})
$$

[note that, comparing this form with definitions (34) and (39) in Section 4 , we have $\beta=\gamma-s]$. In the local approximation, that is, neglecting the integral contribution on the right-hand side of Eq. (66), we look for cylindrically symmetric real solutions $W(\hat{\xi}, \hat{\eta})=W(\rho)(\hat{\xi}=\rho \cos \theta, \hat{\eta}$ $=\rho \sin \theta$ ), which have to satisfy the equation (bright solitons)

$$
\begin{aligned}
\frac{\mathrm{d}^{2} W}{\mathrm{~d} \rho^{2}}+\frac{1}{\rho} \frac{\mathrm{d} W}{\mathrm{~d} \rho} & +\left(1-p^{2}\right) W \\
& -\frac{W}{1+W^{2}}\left[1+\frac{1}{2} \ln \left(1+W^{2}\right)\right]=0,
\end{aligned}
$$

where we have set (see Section 4) $s=1$ and $\beta=p^{2}$ - 1. For dark vortex solitons we look instead for solutions of the kind in which $W(\hat{\xi}, \hat{\eta})=W(\rho) \exp (\operatorname{im} \theta)$, where the real function $W(\rho)$ satisfies the equation

$$
\begin{aligned}
\frac{\mathrm{d}^{2} W}{\mathrm{~d} \rho^{2}}+\frac{1}{\rho} & \frac{\mathrm{d} W}{\mathrm{~d} \rho}-\left(\frac{m^{2}}{\rho^{2}}+1\right) W \\
& +\frac{1+W_{\infty}^{2}}{1+W^{2}} W\left[1+\frac{1}{2} \ln \frac{\left(1+W^{2}\right)}{1+W_{\infty}^{2}}\right]=0,
\end{aligned}
$$

where $m$ is the topological charge and we have taken advantage of the relation (see Section 3) $\gamma=0$, that is, $\beta$ $=1$.

We discuss in Appendix B the numerical procedure that one can follow to obtain the soliton profiles from Eqs. (68) and (69). Here we summarize the results for the soliton existence curves. In particular, we show in Fig. 3 FWHM of the intensity profile versus the peak amplitude for bright solitons and in Fig. 4 the same width versus the background amplitude for a dark vortex soliton with $m$ $=1$. These curves can be compared with the existence curves of the $1+1$-D case (see Fig. 2).

Let us now examine the case of dark solitons without the limitation that $\left|u_{\infty}\right|^{2} \ll 1$. We first note that, after we introduce $g=\sqrt{b} f$, Eq. (55) can be rewritten in the compact form

$$
\hat{\nabla} \cdot\left(\frac{1}{\boldsymbol{Q}} \hat{\nabla} g\right)=-\frac{\partial}{\partial \hat{\eta}}\left(\frac{1}{\boldsymbol{Q}}\right) .
$$

Although Eq. (70) cannot in general be integrated to provide an explicit expression of $g$ in terms of $Q$ to insert into the propagation equation, we can accomplish this program by postulating for $g$ a simple expression corresponding to a reasonable physical model. More precisely,

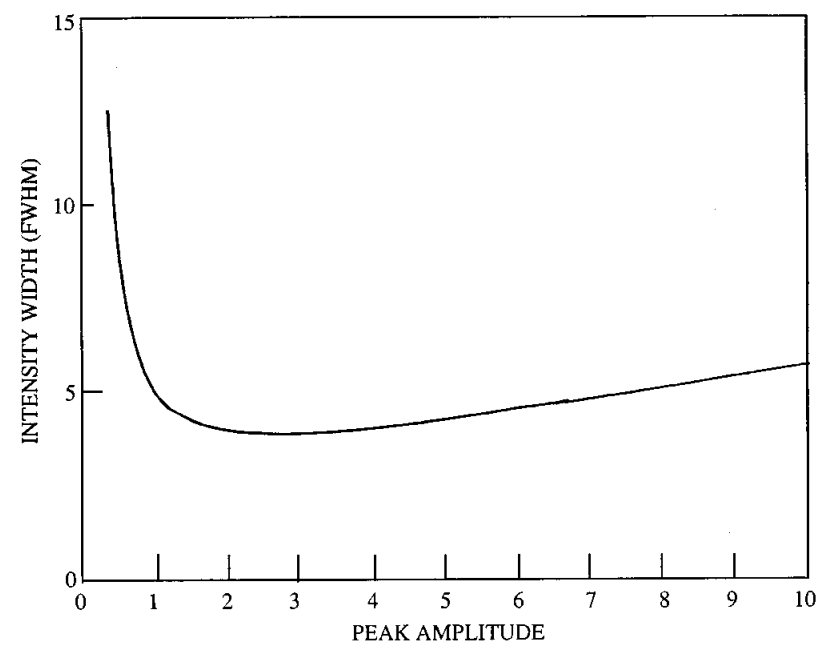

Fig. 3. Existence curve for bright $2+1$-D solitons in the local approximation. The curve is meaningful in the low-intensity regime $|u(0)|^{2} \ll 1$. 


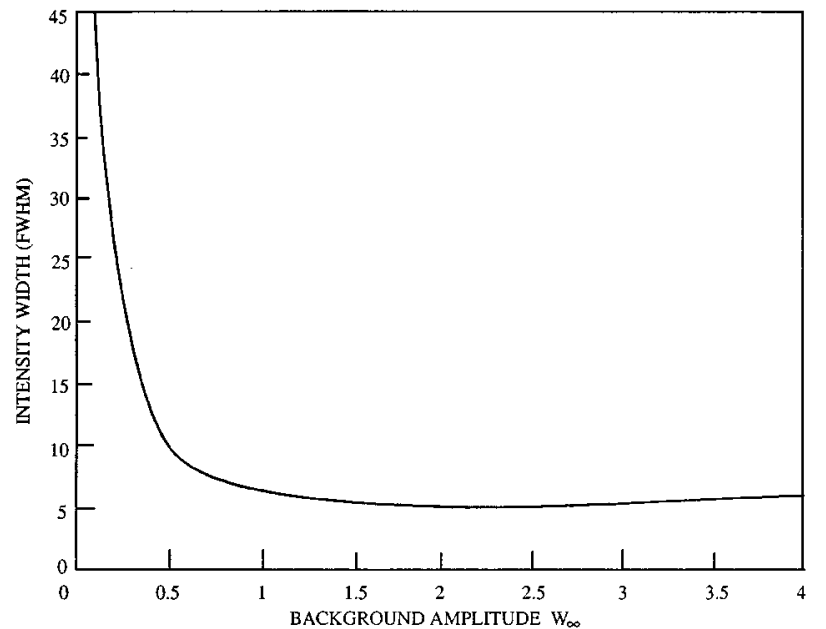

Fig. 4. Existence curve for dark $2+1-\mathrm{D}$ solitons in the local approximation. The curve is meaningful in the low-intensity regime $\left|u_{\infty}\right|^{2} \ll 1$.

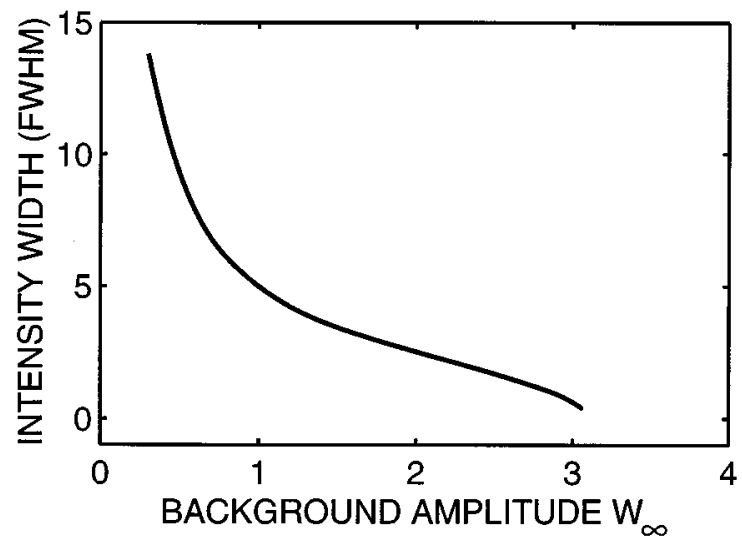

Fig. 5. Existence curve for dark $2+1$-D solitons in the intensity regime $\left|u_{\infty}\right| \simeq 1$.

if we assume that $g(\hat{\xi}, \hat{\eta})=a \rho \sin \theta /\left(b^{2}+\rho^{2}\right)$ (where $a$ and $b$ are two suitable parameters), which exhibits the correct asymptotic dependence for both $\rho \rightarrow 0$ and $\rho \rightarrow \infty, Q$ can easily be expressed in terms of $g$ (see Appendix C). This, in turn, allows us to derive $|u|^{2}=Q$ - 1 , which provides the shape of our soliton, depending on the two parameters $a$ and $b$. We can obtain a first relation between the parameters by imposing that, as $\rho \rightarrow \infty,|u|^{2} \rightarrow\left|u_{\infty}\right|^{2}$; we can derive the second one by inserting $u=W \exp [i(\theta+\hat{\zeta})]$ into propagation equation (65), which, in the stationary case and for dark vortex solitons, takes the form

$$
\begin{aligned}
\frac{\mathrm{d}^{2} W}{\mathrm{~d} \rho^{2}}+\frac{1}{\rho} \frac{\mathrm{d} W}{\mathrm{~d} \rho}- & \frac{1}{\rho 2} W \\
& =\left[1-\left(\frac{1+W_{\infty}^{2}}{1+W^{2}}\right)\left(1+\frac{\partial g}{\partial \hat{\eta}}\right)\right] W,
\end{aligned}
$$

and requiring it to be satisfied for $\rho \rightarrow 0$. Once $a$ and $b$ are derived, we have the explicit expression of the soliton, which, in turn, allows us to obtain the existence curve (see Fig. 5).
It is worthwhile to note that, as expected, the two existence curves for dark solitons coincide for $\left|W_{\infty}\right|^{2} \ll 1$. The main limitation of our model is that it is unable to capture the soliton solution for $\left|W_{\infty}\right|^{2} \geqslant 4$. Covering high-intensity values requires a modification of our simple model.

\section{TIME EVOLUTION TOWARD STEADY STATE}

All the previous developments referred to the asymptotic temporal regime in which the system has reached steady state (i.e., $\partial / \partial t=0$ ). However, it is not difficult, under rather general hypotheses, to draw some conclusions about the evolution of the system toward steady state.

The problem was recently treated for the $1+1-\mathrm{D}$ case,${ }^{56}$ and here we limit ourselves to showing how the temporal evolution of the space-charge field can be described in a simple straightforward way. To this end, let us start by observing that, by taking the partial derivative with respect to time of both sides of Eq. (2) and using Eq. (3), it is possible to deduce the following exact equation:

$$
\hat{\nabla} \cdot \frac{\partial \mathbf{Y}}{\partial \tau}+\hat{\nabla} \cdot(\bar{N} \mathbf{Y})+\sqrt{b} \hat{\nabla}^{2} \bar{N}=0,
$$

where we have introduced the adimensional time $\tau$ $=t / T$, with $T=\tilde{\epsilon} / q \mu \beta_{1}$.

If we now assume that the generation and recombination process reaches steady state much faster than any other process, ${ }^{48}$ we can still take, for times larger than the recombination time, $\partial N_{D}{ }^{+} / \partial t=0$ in Eq. (1), which in turn ensures the validity of Eq. (49). Inserting the corresponding expression for $\bar{N}$ into Eq. (72), we obtain, in the $1+1-\mathrm{D}$ case and in the linear regime for the spacecharge field $(|\tilde{\nabla} \cdot \mathbf{Y}| \ll 1)$, the following equation:

$$
\frac{\partial^{2} Y}{\partial \hat{\xi} \partial \hat{\tau}}+Q \frac{\partial Y}{\partial \hat{\xi}}+Y \frac{\partial Q}{\partial \hat{\xi}}+\sqrt{b} \frac{\partial^{2} Q}{\partial \hat{\xi}^{2}}=0
$$

where $\hat{\tau}=\alpha \tau$. The solution of Eq. (72) with the pertinent boundary conditions reads as follows (note that no approximation is needed, as conversely is assumed in Ref. 56 , on the time scale of variation of $Q$ ):

$$
\begin{aligned}
Y= & -\sqrt{b} \exp \left[-\int^{\hat{\tau}} Q\left(\hat{\xi}, \hat{\tau}^{\prime}\right) \mathrm{d} \hat{\tau}^{\prime}\right] \int^{\hat{\tau}} \frac{\partial Q\left(\hat{\xi}, \hat{\tau}^{\prime}\right)}{\partial \hat{\xi}} \\
& \times \exp \left[\int^{\hat{\tau}^{\prime}} Q\left(\hat{\xi}, \hat{\tau}^{\prime \prime}\right) \mathrm{d} \hat{\tau}^{\prime \prime}\right] \mathrm{d} \hat{\tau}^{\prime} \\
& +Y_{0} \exp \left[-\int^{\hat{\tau}} Q\left(\hat{\xi}, \hat{\tau}^{\prime}\right) \mathrm{d} \hat{\tau}^{\prime}\right] \\
& \times \int_{0}^{\hat{\tau}} \exp \left[\int^{\hat{\tau}^{\prime}} Q\left(\hat{\xi}, \hat{\tau}^{\prime \prime}\right) \mathrm{d} \hat{\tau}^{\prime \prime}\right] \mathrm{d} \hat{\tau}^{\prime} \\
& +Y_{0} \exp \left[-\int^{\hat{\tau}} Q\left(\hat{\xi}, \hat{\tau}^{\prime}\right) \mathrm{d} \hat{\tau}^{\prime}\right] .
\end{aligned}
$$

Equation (74) shows that the steady-state regime relative to the screening solitons is actually reached for $\hat{\tau}>1 /(1$ 
$\left.+\left\langle|u|^{2}\right\rangle\right)$, where the angle brackets stand for a time average, that is, for time $t$ larger than the dielectric relaxation time ${ }^{48} T_{\mathrm{di}}=T /\left(1+\left\langle|u|^{2}\right\rangle\right)$.

\section{CONCLUSIONS}

In this paper we have considered the problem of threedimensional $(2+1-\mathrm{D})$ propagation of an optical beam in a biased photorefractive crystal in the asymptotic temporal regime in which the screening of the external static field that is due to the photoexcited charges has already taken place. In particular, we have examined the conditions under which it should be possible to propagate selftrapped beam configurations (bright or dark solitons). To place into evidence the basic differences between twoand three-dimensional cases, we briefly recalled the ( 1 +1 -D) approach used in the former situation and then examined in full generality the $(2+1$-D) case. The main result of the paper is the derivation of a system of coupled equations that completely describes nonlinear propagation in photorefractive media.

In the low-intensity regime the nonlinear interaction term contains a local symmetric contribution that can support circular solitons. The study of the propagation equation in the presence of that contribution alone provides, in particular, the soliton existence curves, that is, the relation between the amplitude and the width of the input beam at $z=0$ that has to be fulfilled if the influence of the asymmetric nonlocal part of the interaction is to play a negligible distorting role in its $z$ evolution over the crystal length.

For the low-intensity regime we indeed have numerically found that both bright and dark initially circular beams self-trap but also evolve into elliptical beams after a propagation distance of roughly one diffraction length (in normalized units). The implication is that we do not find solitons in the low-intensity regime, a conclusion that agrees with the available published experimental results: All existing experiments on self-trapping of $2+1-\mathrm{D}$ screening solitons have been performed at $\left|u_{0}\right|^{2},\left|u_{\infty}\right|^{2}$ larger than or equal to 1 . In fact, for peak intensity lower than 0.5 the beam was found to be unstable, varying with time and never reaching steady state. ${ }^{30}$

In the case of intermediate and high intensity, the approximations used for low intensity are no longer valid, and the problem of solving the stationary equations for the soliton profile is much more complicated. However, for dark solitons only, we found a simplified method to obtain approximate solutions for background intensity values up to 4 (in normalized units). These results, even if they already show the potential of the method, are merely preliminary and have to be extended to cover other regimes for both bright and dark solitons.

\section{APPENDIX A}

Let us first prove the asymptotic behavior [relation (45)]. To this aim, we recall the following textbook results. If $\bar{N}(\hat{\xi}, \hat{\eta})$ is a positive-definite bounded function that satisfies asymptotic condition (44), and if $f(\hat{\xi}, \hat{\eta})$ vanishes as $\rho \rightarrow \infty$ sufficiently fast that its integral

$$
C=\int_{-\infty}^{+\infty} \mathrm{d} \hat{\xi} \int_{-\infty}^{+\infty} \mathrm{d} \hat{\eta} f(\hat{\xi}, \hat{\eta})
$$

is finite, then the solution $\psi(\hat{\xi}, \hat{\eta})$ of the equation

$$
\hat{\nabla}_{\perp} \cdot\left(\bar{N} \hat{\nabla}_{\perp} \psi\right)=f,
$$

with the boundary condition $(\hat{\xi}=\rho \cos \theta, \hat{\eta}=\rho \sin \theta)$

$$
\lim _{\rho \rightarrow \infty} \frac{1}{\rho} \psi(\hat{\xi}, \hat{\eta})=0,
$$

exists and is unique (up to an additive constant). Moreover, the asymptotic behavior of $\psi$, as $\rho \rightarrow \infty$, is

$$
\psi(\hat{\xi}, \hat{\eta}) \rightarrow a \ln \rho+O(1 / \rho)
$$

where the asymptotic constant $a$ takes the value

$$
a=\frac{C}{2 \pi \bar{N}_{\infty}},
$$

where $\bar{N}_{\infty}$ is given by asymptotic condition (44). In our case [see Eq. (41)], $f=-\partial \bar{N} / \partial \hat{\xi}$, so the integral $C$ vanishes; $C=0$. Hence Eq. (A5) and relation (A4) imply relation (38) and, because of Eq. (32), the asymptotic behavior in relation (45).

Let us now consider Eq. (40). Because of the result just proved, namely, that $\psi=O(1 / \rho)$ as $\rho \rightarrow \infty$, Eq. (40) is the stationary Schrödinger equation with a potential that is $O\left(1 / \rho^{2}\right)$ as $\rho \rightarrow \infty$. Let $W$ be the bright soliton solution (if it exists); i.e., let us assume that

$$
W \rightarrow 0 \quad \text { as } \rho \rightarrow \infty,
$$

and set $W=|W| \exp (i \varphi)$, where $\varphi(\hat{\xi}, \hat{\eta})$ is the phase of the soliton. Then Eq. (40) immediately splits into the two real equations:

$$
\begin{gathered}
\hat{\nabla}_{\perp}{ }^{2}|W|=\left[\gamma+\left(\hat{\nabla}_{\perp} \varphi\right)^{2}+s \frac{\partial \psi}{\partial \hat{\xi}}\right]|W|, \\
|W| \hat{\nabla}_{\perp}{ }^{2} \varphi+2 \hat{\nabla}_{\perp}|W| \cdot \hat{\nabla}_{\perp} \varphi=0 .
\end{gathered}
$$

Equation (A8) can equivalently be rewritten as

$$
\hat{\nabla}_{\perp} \cdot\left(|W|^{2} \hat{\nabla}_{\perp} \varphi\right)=0
$$

which implies that

$$
\hat{\nabla}_{\perp} \cdot\left(|W|^{2} \varphi \hat{\nabla}_{\perp} \varphi\right)=|W|^{2}\left(\hat{\nabla}_{\perp} \varphi\right)^{2} .
$$

If we integrate both sides of Eq. (A10) on the whole plane, and use the Gauss divergence theorem together with vanishing condition (A6) and the assumption that the phase $\varphi$ is single valued, we obtain

$$
\int_{-\infty}^{+\infty} \mathrm{d} \hat{\xi} \int_{-\infty}^{+\infty} \mathrm{d} \hat{\eta}|W|^{2}\left(\hat{\nabla}_{\perp} \varphi\right)^{2}=0
$$

with the obvious implication that the soliton phase $\varphi$ is constant, i.e., relation (47).

Let us turn our attention to the dark soliton, whose phase $\varphi$ satisfies asymptotic condition (47), where $\varphi_{\infty}(\hat{\xi}, \hat{\eta})$ is the asymptotic phase of the soliton. The leading terms in Eqs. (A7) and (A9) in the limit $\rho \rightarrow \infty$ lead to the following equations for the phase function $\varphi_{\infty}$ :

$$
\left(\hat{\nabla}_{\perp} \varphi_{\infty}\right)^{2}=p^{2}, \quad \hat{\nabla}_{\perp}^{2} \varphi_{\infty}=0
$$


where $p^{2}=-\gamma>0$ and the right-hand side is the leading term. The second of Eqs. (A12) shows that $\varphi_{\infty}$ is harmonic; the first one implies that $\varphi_{\infty}$ cannot diverge faster than linearly as $\rho \rightarrow \infty$. Therefore the general behavior of $\varphi_{\infty}$ has to be (if we omit an additional constant)

$$
\begin{aligned}
& \varphi_{\infty}= p(\hat{\xi} \sin \delta+\hat{\eta} \cos \delta)+c_{1} \ln \rho+c_{2} \theta \\
&+O(1 / \rho) \quad \text { as } \quad \rho \rightarrow \infty,
\end{aligned}
$$

where $\delta, c_{1}$, and $c_{2}$ are constants. By integrating the left-hand side of Eq. (A9) over the domain $\rho \leqslant R$, and by using Gauss divergence theorem, we can write

$$
R \int_{0}^{2 \pi} \mathrm{d} \theta|W|^{2} \partial \varphi / \partial \rho=0,
$$

which, in the limit $R \rightarrow \infty$ [and taking into account asymptotic formulas (47) and (A13)], yields

$$
c_{1}=0 \text {. }
$$

Finally, asymptotic expression (48) follows when we note that the parameter $c_{2}$ in Eq. (A13) has to be an integer, say, $c_{2}=m$, because $W$, and therefore $\left|W_{\infty}\right| \exp \left(i \varphi_{\infty}\right)$, is a single-valued function.

These findings are simplified if Eq. (A7) is replaced by its local approximation:

$$
\hat{\nabla}_{\perp}|W|+\left[-p^{2}+\left(\hat{\nabla}_{\perp} \varphi\right)^{2}-\frac{s}{2} \ln \frac{1+|W|^{2}}{1+\left|W_{\infty}\right|^{2}}\right]|W|,
$$

together with the condition that the amplitude $|W|$ be $\theta$ independent (cylindrically symmetric dark soliton). According to the general result given above [see Eqs. (A13) and (A15)], we set

$$
\varphi=p \rho \sin (\theta+\delta)+m \theta+\alpha(\rho, \theta),
$$

where the function $\alpha$ is asymptotically vanishing, i.e., $\alpha$ $=0(1 / \rho)$ as $\rho \rightarrow \infty$. Consider now that the $\theta$ derivative of $\left(\hat{\nabla}_{\perp} \varphi\right)^{2}$ vanishes; therefore, because of Eq. (A17), we get

$$
\lim _{\rho \rightarrow \infty}\left[\rho \frac{\partial}{\partial \theta}\left(\hat{\nabla}_{\perp} \varphi\right)^{2}\right]=-2 m p \sin (\theta+\delta)=0,
$$

which implies that $p=0$ for vortex solitons $(m \neq 0)$. We note next that the function $\alpha$ that appears in Eq. (A17) satisfies Eq. (A8) (i.e., with $\varphi$ being replaced by $\alpha$ ); therefore integrating Eq. (A10), with $\varphi$ being replaced by $\alpha$, yields the equation $\left(\alpha_{\rho} \equiv \partial \alpha / \partial \rho\right.$ )

$$
\begin{aligned}
& R|W(R)|^{2} \int_{0}^{2 \pi} \mathrm{d} \theta \alpha(R, \theta) \alpha_{\rho}(R, \theta) \\
&=\int_{0}^{R} \mathrm{~d} \rho \rho|W(\rho)|^{2} \int_{0}^{2 \pi} \mathrm{d} \theta\left(\hat{\nabla}_{\perp} \alpha\right)^{2},
\end{aligned}
$$

which, because of the asymptotic vanishing of $\alpha$ as $\rho \rightarrow \infty$, implies that $\alpha=0$ and therefore that $\varphi=m \theta$.

\section{APPENDIX B}

Here we briefly describe the way to integrate ordinary differential equation (60), which, for bright solitons, specializes to

$$
\frac{\mathrm{d}^{2} W}{\mathrm{~d} \rho^{2}}+\frac{1}{\rho} \frac{\mathrm{d} W}{\mathrm{~d} \rho}=-\frac{\mathrm{d} V(W)}{\mathrm{d} W},
$$

where the potential function $V(W)$ is

$$
\begin{aligned}
V(W)= & 1 / 2\left(1-p^{2}\right) W^{2}-1 / 2 \ln \left(1+W^{2}\right) \\
& -1 / 8 \ln ^{2}\left(1+W^{2}\right) .
\end{aligned}
$$

In analogy with classical mechanics, Eq. (B1) is the equation of motion of a particle in the potential [Eq. (B2)] with the friction term $(1 / \rho) \mathrm{d} W / \mathrm{d} \rho$. Near the initial time $\rho$ $=0$ there is only one regular solution, which is an even function of $\rho$ and depends only on the initial position $W(0) \equiv a$ according to the Taylor expansion

$$
\begin{aligned}
W(\rho)= & a\left(1+\frac{\rho^{2}}{4}\left\{p^{2}+\left[1 / 2 \ln \left(1+a^{2}\right)-a^{2}\right] /\right.\right. \\
& \left.\left.\left(1+a^{2}\right)\right\}\right)+O\left(\rho^{4}\right) .
\end{aligned}
$$

For a generic value of $a$ this solution will eventually converge with damped oscillations to one of the two values $\pm W_{m}$ where the potential [Eq. (B2)] takes its minimum [i.e., $V^{\prime}\left(W_{m}\right)=0$ ]. However, the function $W(\rho)$ will stay positive only for a special value $a=\bar{a}$, if $\bar{a}>0$, and will asymptotically decay to zero with the law $\exp (-p \rho) / \sqrt{\rho}$ as $\rho \rightarrow \infty$. This solution yields the bright soliton profile that interests us. To compute the amplitude $\bar{a}$ and plot it against its width $l_{s}$, we proceed as follows.

For a given value of the parameter $p$, to avoid the $\rho$ $=0$ singularity we integrate Eq. (B1) with the initial conditions [see Eq. (B3)] that

$$
\begin{aligned}
W(\epsilon) & =a \\
\frac{\mathrm{d} W}{\mathrm{~d} \rho}(\epsilon) & =1 / 2 \epsilon a\left\{p^{2}+\left[1 / 2 \ln \left(1+a^{2}\right)-a^{2}\right] /\left(1+a^{2}\right)\right\}
\end{aligned}
$$

at a small value of $\epsilon$, e.g., $\epsilon=10^{-8}$. Then, as in the standard shooting method of solving boundary-value problems, we adjust the value of $a$ to match the vanishing asymptotic limit as $\rho \rightarrow+\infty$. Once this value of $a$ say, $a=\bar{a}(p)$, is found, we calculate the width of the soliton. We might define the width $l_{s}(p)$ as either the positive zero of the second derivative (i.e., $\mathrm{d}^{2} W / \mathrm{d} \rho^{2}=0$ at $\rho$ $\left.=l_{s} / 2\right)$ or the usual FWHM intensity width, namely, $W^{2}\left(\rho=l_{s} / 2\right)=A^{2} / 2$, where $A$ is the maximum of $W(\rho)$. Finally, we obtain the soliton existence curve by plotting $l_{s}$ against the peak amplitude $A$ or the peak intensity $A^{2}$.

Next we consider the integration of Eq. (60) when it models the dark soliton. In this case the parameters are $\gamma=0$ and $s=-1$, and the phase of the field profile $W$ is no longer vanishing:

$$
\begin{aligned}
W(\rho, \theta) & =v(\rho) \exp (i m \theta), \quad m= \pm 1, \pm 2, \ldots, \\
v & =v^{*}
\end{aligned}
$$

and the equation that we have to integrate reads as

$$
\frac{\mathrm{d}^{2} v}{\mathrm{~d} \rho^{2}}+\frac{1}{\rho} \frac{\mathrm{d} v}{\mathrm{~d} \rho}-m^{2} \frac{v}{\rho^{2}}=-\frac{\mathrm{d} V(v)}{\mathrm{d} v},
$$

where the potential $V=V(v)$ now takes the expression 


$$
\begin{aligned}
V(v)= & -1 / 2 v^{2}+1 / 2 \ln \left(1+v^{2}\right) \\
& \times\left[1+v_{\infty}{ }^{2}+1 / 4 \ln \left(1+v^{2}\right)-1 / 2 \ln \left(1+v_{\infty}{ }^{2}\right)\right]
\end{aligned}
$$

and $v_{\infty} \equiv\left|W_{\infty}\right|$ is the asymptotic constant value of $v$ (i.e., $v \rightarrow v_{\infty}$ as $\left.\rho \rightarrow \infty\right)$. Again, for each value of $m \neq 0$ there is only one regular solution of Eqs. (B6) and (B7) at $\rho$ $=0$, and its behavior is

$$
\begin{aligned}
v(\rho)= & a \rho^{m}\left\{1-\frac{\rho^{2}}{8(m+1)}\right. \\
& \left.\times\left[\left(1+v_{\infty}^{2}\right) \ln \left(1+v_{\infty}^{2}\right)-2 v_{\infty}^{2}\right]+O\left(\rho^{4}\right)\right\},
\end{aligned}
$$

where $a$ is an arbitrary constant. By keeping the analogy with classical mechanics, Eq. (B6) shows the existence of a force $\propto \rho^{-2}$ in addition to the friction and the potential force. Therefore it follows that, only for a particular (say, positive) value $a=\bar{a}$ [see Eq. (B8)], the particle moves to the right and eventually stops for $\rho \rightarrow \infty$ at the unstable equilibrium position $v=v_{\infty}$.

Moreover, it is easily seen that the value $v_{0}$ is attained from below with the asymptotic behavior

$$
v(\rho)=v_{\infty}\left[1-\left(1+\frac{1}{v_{\infty}^{2}}\right) \frac{m^{2}}{\rho^{2}}+O\left(\frac{1}{\rho^{4}}\right)\right] .
$$

If, instead, $0<a<\bar{a}, v(\rho)$ will eventually reach the origin (stable position $v=0$ ) with damped oscillations; or, if $a>\bar{a}$, the solution will run away to infinity. Thus the method of solution goes as before. For a given integer $m \neq 0$, an asymptotic value $v_{\infty}$, and a small $\epsilon$ (e.g., $\epsilon$ $=10^{-8}$ ), we integrate Eq. (B6) with the initial conditions

$$
v(\epsilon)=a, \quad \frac{\mathrm{d} v}{\mathrm{~d} \rho}(\rho=\epsilon)=m a \epsilon^{m-1}
$$

for a trial value $a$, and then we search for the value $a$ $=\bar{a}$ by requiring that the corresponding solution $v(\rho)$ match the asymptotic behavior in Eq. (B9). The resulting solution $v=v(\rho)$ is the profile of the dark soliton, and we obtain the relevant information by plotting $l_{s}$ against $v_{\infty}$, where $l_{s}$ is the soliton width [at either the zero of the second derivative or the FWHM intensity width $v^{2}(\rho$ $\left.\left.=l_{s} / 2\right)=v_{\infty}^{2} / 2\right]$.

\section{APPENDIX C}

Here we solve Eq. (70) with respect to $Q$ [see Eq. (50)] for a special choice of the function [see Eqs. (53) and (24)]:

$$
g(\hat{\xi}, \hat{\eta})=\sqrt{b} f(\xi, \eta),
$$

namely, $g(\hat{\xi}, \hat{\eta})=\rho G\left(\rho^{2}\right) \sin \theta$, with

$$
G(z)=a /\left(b^{2}+z\right) \text {. }
$$

Indeed, in this case, Eq. (70) is a first-order linear differential equation for $C(z)$ :

$$
C(z)=1 / Q(\rho), \quad z=\rho^{2},
$$

which reads $\left({ }^{\prime} \equiv \mathrm{d} / \mathrm{d} z\right)$ as

$$
\left[\left(1+G+2 z G^{\prime}\right) C\right]^{\prime}+G^{\prime} C=0 .
$$

Integrating Eq. (C4) with the initial condition $C(0)=1$ (which is appropriate for dark solitons) yields the expression

$$
\begin{aligned}
C(z)= & \frac{[1+G(0)]}{1+G(z)+2 z G^{\prime}(z)} \\
& \times \exp \left[-\int_{0}^{z} \mathrm{~d} t \frac{G^{\prime}(t)}{1+G(t)+2 t G^{\prime}(t)}\right] .
\end{aligned}
$$

Our choice, Eq. (C2), is simple enough to allow the integral on the right-hand side to be computed easily, so we can arrive at the explicit expression $\left(m \equiv b^{2} / a\right)$

$C(z)$

$$
\begin{aligned}
& =\frac{(1+m)(m+z / a)^{2}}{m\left[(m+z / a)^{2}+m-z / a\right]} \\
& \times\left[\frac{2 m(1+m)+(2 m-1+\sqrt{1-8 m}) z / a}{2 m(1+m)+(2 m-1-\sqrt{1-8 m}) z / a}\right]^{1 / \sqrt{1-8 m}},
\end{aligned}
$$

which finally provides the soliton intensity profile:

$$
|u(\rho)|^{2}=\frac{1}{C\left(\rho^{2}\right)}-1 .
$$

\section{ACKNOWLEDGMENTS}

B. Crosignani gratefully acknowledges the support of collaborative NATO research grant 930240. M. Segev gratefully acknowledges the support of the National Science Foundation.

The research of S. Trillo was carried out under the agreement between Fondazione Ugo Bordoni and the Italian Post and Telecommunication Administration.

\section{REFERENCES}

1. R. Y. Chiao, E. Garmire, and C. H. Townes, Phys. Rev. Lett. 13, 479 (1964).

2. V. E. Zakharov and A. B. Shabat, Sov. Phys. JETP 34, 62 (1972); 37, 823 (1973).

3. J. E. Bjorkholm and A. Ashkin, Phys. Rev. Lett. 32, 129 (1974).

4. A. Barthelemy, S. Maneuf, and C. Froehly, Opt. Commun. 55, 201 (1985)

5. J. S. Aitchison, A. M. Weiner, Y. Silberberg, M. K. Oliver, J. L. Jackel, D. E. Leaird, E. M. Vogel, and P. W. Smith, Opt. Lett. 15, 471 (1990).

6. P. L. Kelley, Phys. Rev. Lett. 15, 1005 (1965).

7. V. E. Zakharov and A. M. Rubenchik, JETP 38, 494 (1974).

8. A. W. Snyder, D. J. Mitchell, L. Poladian, and F. Ladouceur, Opt. Lett. 16, 21 (1991).

9. S. Gatz and J. Herrmann, J. Opt. Soc. Am. B 8, 2296 (1991)

10. J. M. Soto-Crespo, D. R. Heatley, E. M. Wright, and N. N. Akhmediev, Phys. Rev. A 44, 636 (1991).

11. Y. Chen, Opt. Lett. 16, 4 (1991).

12. M. Karlsson, Phys. Rev. A 46, 2726 (1992).

13. A. Kumar, T. Kurz, and W. Lauterborn, Phys. Rev. A 53, 1166 (1996).

14. V. Tikhonenko, J. Christou, and B. Luther-Davies, Phys. Rev. Lett. 76, 2698 (1996).

15. W. E. Torruellas, Z. Wang, D. J. Hagan, E. W. Van Stryland, G. I. Stegeman, L. Torner, and C. R. Menyuk, Phys. Rev. Lett. 74, 5036 (1995).

16. M. Segev, B. Crosignani, A. Yariv, and B. Fischer, Phys. Rev. Lett. 68, 923 (1992). 
17. B. Crosignani, M. Segev, D. Engin, P. DiPorto, and A. Yariv, J. Opt. Soc. Am. B 10, 449 (1993).

18. D. N. Christodoulides and M. I. Carvalho, Opt. Lett. 19, 714 (1994).

19. G. Duree, J. L. Shultz, G. Salamo, M. Segev, A. Yariv, B. Crosignani, P. DiPorto, E. Sharp, and R. Neurgaonkar, Phys. Rev. Lett. 71, 533 (1993).

20. M. Segev, A. Yariv, B. Crosignani, P. DiPorto, G. Duree, G. Salamo, and E. Sharp, Opt. Lett. 19, 1296 (1994).

21. G. Duree, G. Salamo, M. Segev, A. Yariv, B. Crosignani, P. DiPorto, and E. Sharp, Opt. Lett. 19, 1195 (1994).

22. G. Duree, M. Morin, G. Salamo, M. Segev, A. Yariv, B. Crosignani, and P. DiPorto, Phys. Rev. Lett. 74, 1978 (1995)

23. M. Morin, G. Duree, G. Salamo, and M. Segev, Opt. Lett. 20, 2066 (1995).

24. M. Segev, G. C. Valley, B. Crosignani, P. DiPorto, and A. Yariv, Phys. Rev. Lett. 73, 3211 (1994).

25. D. N. Christodoulides and M. I. Carvalho, J. Opt. Soc. Am. B 12, 1628 (1995).

26. S. R. Singh and D. N. Christodoulides, Opt. Commun. 118, 569 (1995).

27. M. Segev, M. Shih, and G. C. Valley, J. Opt. Soc. Am. B 13, 706 (1996).

28. Observations of steady-state self-focusing effects were first reported by M. D. Iturbe-Castillo, P. A. Marquez-Aguilar, J. J. Sánchez-Mondragón, S. Stepanov, and V. Vysloukh, Appl. Phys. Lett. 64, 408 (1994).

29. M. Shih, M. Segev, G. C. Valley, G. Salamo, B. Crosignani, and P. DiPorto, Electron. Lett. 31, 826 (1995).

30. M. Shih, P. Leach, M. Segev, M. Garrett, G. Salamo, and G. C. Valley, Opt. Lett. 21, 324 (1996).

31. M. Shih, M. Segev, and G. Salamo, Opt. Lett. 21, 931 (1996).

32. M. Shih and M. Segev, Opt. Lett. 21, 1538 (1996).

33. K. Kos, H. Meng, G. Salamo, M. Shih, M. Segev, and G. C. Valley, Phys. Rev. E 53, R4330 (1996).

34. M. Chauvet, S. A. Hawkins, G. Salamo, M. Bryant, M. Segev, D. F. Bliss, and G. Bryant, Opt. Lett. 16, 1333 (1996).

35. Z. Chen, M. Mitchell, M. Shih, M. Segev, M. Garrett, and G. C. Valley, Opt. Lett. 21, 629 (1996).

36. Z. Chen, M. Mitchell, and M. Segev, Opt. Lett. 21, 716 (1996).
37. M. D. Iturbe-Castillo, J. J. Sánchez-Mondragón, S. I. Stepanov, M. B. Klein, and B. A. Wechsler, Opt. Commun. 118, 515 (1995).

38. M. Segev, G. C. Valley, S. R. Singh, M. I. Carvalho, and D. N. Christodoulides, Opt. Lett. 20, 1764 (1995).

39. S. R. Singh, M. I. Carvalho, and D. N. Christodoulides, Opt. Lett. 20, 2177 (1995).

40. M. I. Carvalho, S. R. Singh, and D. N. Christodoulides, Phys. Rev. E 53, R53 (1995).

41. W. Krolikowski, N. Akhmedhiev, and B. Luther-Davies, Opt. Lett. 21, 782 (1996).

42. D. N. Christodoulides, S. R. Singh, M. I. Carvalho, and M. Segev, Appl. Phys. Lett. 68, 1763 (1996).

43. Z. Chen, M. Segev, T. H. Coskun, and D. N. Christodoulides, Opt. Lett. 21, 1436 (1996).

44. G. C. Valley, M. Segev, B. Crosignani, A. Yariv, M. M. Fejer, and M. C. Bashaw, Phys. Rev. A 50, R4457 (1994).

45. M. Taya, M. C. Bashaw, M. M. Fejer, M. Segev, and G. C. Valley, Phys. Rev. A 52, 3095 (1995).

46. M. Taya, M. C. Bashaw, M. M. Fejer, M. Segev, and G. C. Valley, Opt. Lett. 21, 943 (1996).

47. P. Gunter and J.-P. Huignard, Photorefractive Materials and Their Applications (Springer, New York, 1988), Vols. I and II.

48. P. Yeh, Introduction to Photorefractive Nonlinear Optics (Wiley, New York, 1993).

49. A. A. Zozulya and D. Z. Anderson, Phys. Rev. A 51, 1520 (1995).

50. D. Burak and W. Nasalski, Appl. Opt. 33, 6393 (1994).

51. A. L. Fetter, Phys. Rev. 151, 100 (1966).

52. G. S. McDonald, U. S. Syed, and W. J. Firth, Opt. Commun. 94, 469 (1992).

53. G. A. Swartzlander and C. T. Law, Phys. Rev. Lett. 69, 2503 (1992).

54. N. Korneev, P. A. Marquez Aguilar, J. J. Sánchez Mondragón, S. Stepanov, M. Klein, and B. Wechsler, J. Mod. Opt. 43, 311 (1996).

55. See, e.g., G. P. Agrawal, Nonlinear Fiber Optics (Academic, New York, 1989).

56. N. Fressengeas, J. Maufoy, and G. Kugel, Phys. Rev. E 54, 6866 (1996). 